\title{
A Rare Presentation of Cavernous Hemangioma of Both Inferior Turbinates
}

\author{
KN Salimath, ${ }^{1}$ N Ramakrishnan, ${ }^{2}$ JR Galagali ${ }^{2}$
}

\author{
Introduction \\ The nasal cavity presents with various types of neoplasms, including epithelial and mesenchymal tumors. Cavernous hemangioma \\ of nasal cavity is quite rare. It usually presents as a unilateral mass arising from mucosa of nasal cavity. \\ Case Report \\ We describe here, a case of 25-year-old serving soldier, who was referred to our hospital with a mass in both nasal cavities \\ with bilateral nasal obstruction. On examination, the mass originated from the inferior aspect of inferior turbinate of both \\ sides. Subsequently on histological examination after complete endoscopic excision revealed that the mass was a cavernous \\ hemangioma arising from both inferior turbinates. \\ Discussion \\ To our knowledge, this is the first case of cavernous hemangioma arising from both inferior turbinate reported in literature. \\ Keywords \\ Hemangioma, Cavernous; Nose Neoplasms
}

$\underline{\text { ABSTRACT }}$

$\mathrm{H}$ emangioma is a benign neoplasm that originates in the vascular tissue of skin, mucosa, bone, muscles and glands. ${ }^{1}$ However it is usually located on the skin or oral mucosa, but its occurrence in the nasal cavity and paranasal sinuses is rare. Hemangioma comprises of about $20 \%$ of all benign neoplasms of the nasal cavity. Hemangioma of the nasal cavity occurs most commonly on the septum (65\%), lateral wall $(18 \%)$, and vestibule $(16 \%) .{ }^{2}$ Hemangioma are classified histologically according to the predominant type of vascular channel as capillary, cavernous, or mixed. This disease usually presents as unilateral epistaxis with nasal obstruction. To our knowledge, cavernous hemangioma involving both of inferior turbinates has not been reported in literature. Here we report a case of cavernous hemangioma arising from both of the inferior turbinates, successfully treated by trans-nasal endoscopic excision technique.

\section{Case report}

A 25 year old serving soldier referred to Dept of Otolaryngology with complaints of progressive nasal obstruction both side of six months duration and associated with mouth breathing. There was no history of epistaxis or facial trauma. Anterior rhinoscopic examination revealed a pedunculated mass with thin stalk arising from inferior aspect of both inferior turbinates. Endoscopic examination revealed a smooth polypoid, greyish mass arising from inferior aspect of inferior turbinates bilaterally compromising airway of both nostrils (Fig. 1).

The mass appears to be distinctly separate and hanging from inferior aspect of both inferior turbinates with pedicle. The mass was extending posteriorly till posterior choanae on both sides. Non contrast CT scan showed a soft tissue density arising from inferior turbinates, extending till posterior choanae without bony erosion (Fig-2).

Past history revealed that the patient had undergone an operative procedure for nasal blockade in Apr 2007. Details of correct diagnosis and procedure were not known. After the previous operation, he was

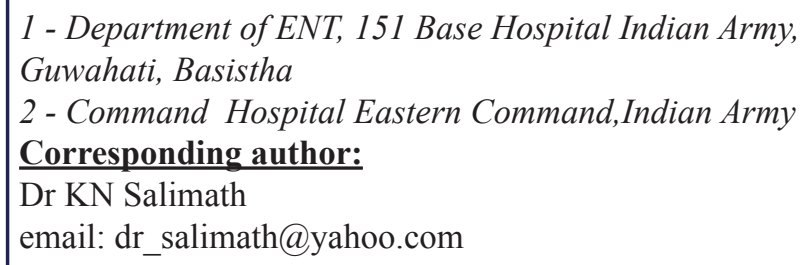




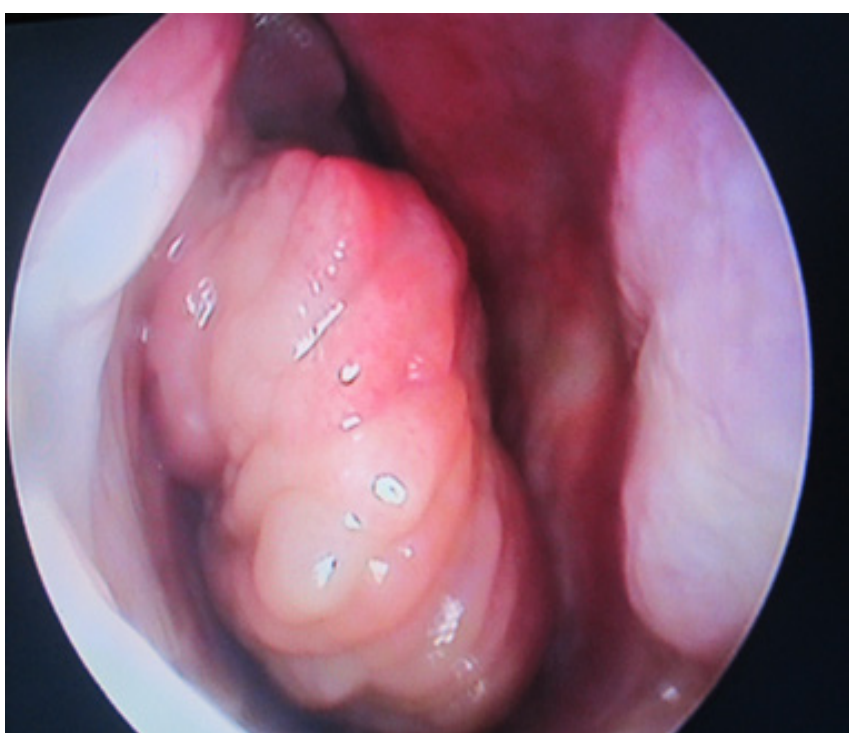

Fig. 1. Endoscopic examination: Smooth polypoid, greyish mass arising from inferior aspect of inferior turbinate

asymptomatic till six months back. Initial examination and the radiological assessment suggested that the lesion was a benign tumor. The patient underwent planned tumor excision via endonasal endoscopic approach. Complete wide resection of the tumor was achieved by transnasal endoscopic surgery and bleeding was controlled with coagulation suction and anterior

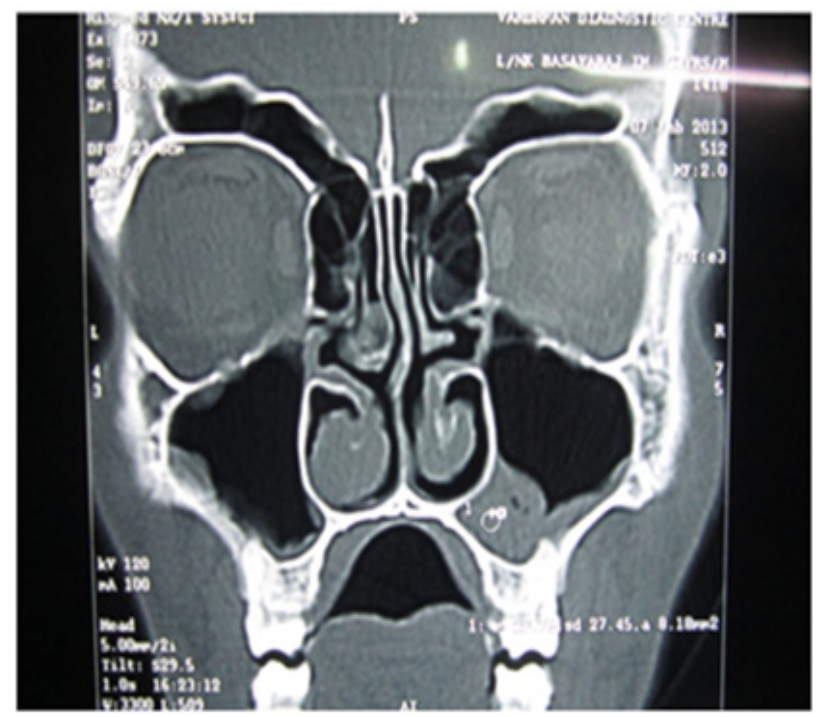

Fig. 2. NCCT showing a soft tissue density arising from inferior turbinates nasal packing. During the immediate post operative period, patient had massive nasal bleeding. Patient was reintubated and nasal pack removed to access bleeding site, there was diffuse oozing and bleeding controlled with posterior and anterior nasal pressure packing. Blood transfusion was done for hypotension. Nasal pack removed after 48 hours and patient recovered well.

Macroscopically, the tumor was $3 \times 5 \mathrm{~cm}$ in size with intact mucosa (Fig-3).

Microscopically, the tumor showed hemorrhagic polypoidal tissue with the presence of anastomosing blood vessels of variable sizes, lined with flattened endothelial cells. The histological diagnosis was cavernous hemangioma (Figs. 4 \& 5).

The patient was followed up six monthly for three years and endoscopic examination showed no recurrence.

\section{Discussion}

Hemangioma is a benign tumor originating in the vascular tissue of mucosa, skin, muscles, glands, and bones. Head and neck is a common region for hemangioma. Hemangioma of the nasal cavity is extremely rare. The most common site for nasal hemangioma is nasal septum,

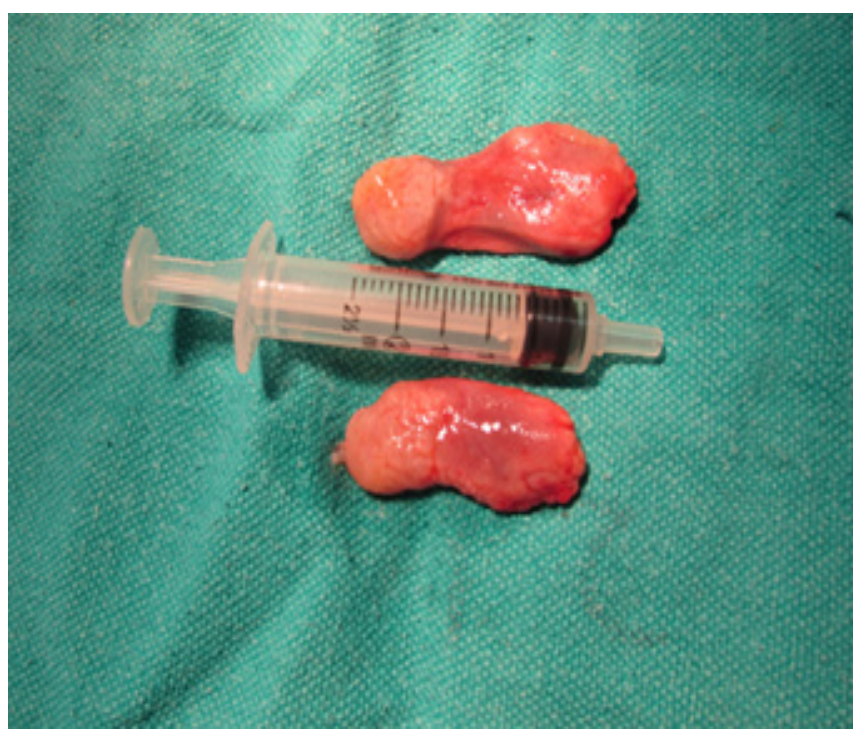

Fig. 3. The Tumor specimen $3 \times 5 \mathrm{~cm}$ in size with intact mucosa. 


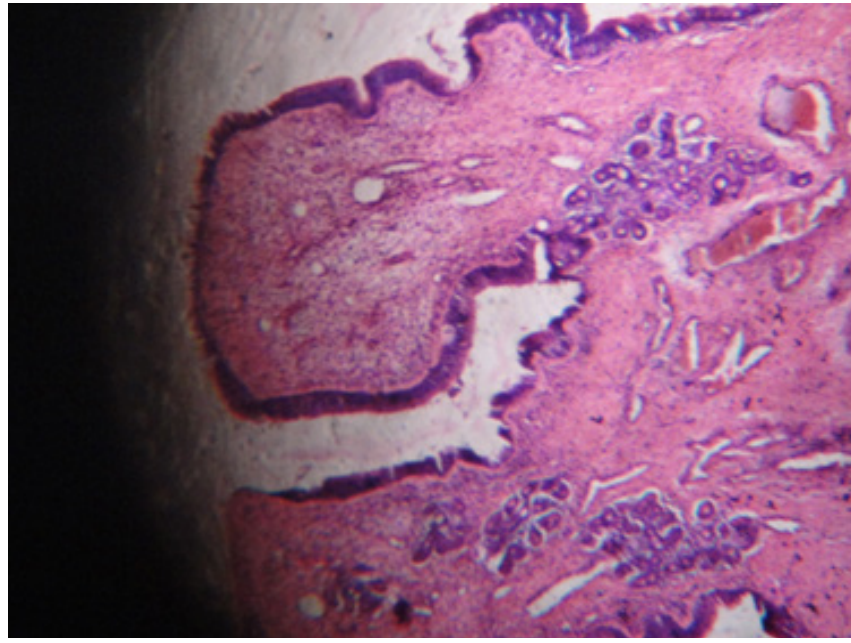

Fig. 4. Hematoxylin and eosin (H\&E, 10X).

followed by the lateral wall and vestibule. ${ }^{1}$ Many reports have shown a hemangioma arising in the turbinate and most of them arise from the mucosa. ${ }^{2}$ Hemangioma is classified histologically as capillary, cavernous or mixed according to predominant vascular channels. The subtypes also vary in their clinical features. Capillary hemangiomas are more frequent than cavernous and usually present at a younger age and may involute spontaneously. In contrast, cavernous hemangiomas are very rare and typically seen in adults. They do not undergo spontaneous involution and are more likely to cause compression of surrounding structures. The lack of feeding vessels to capillary hemangiomas makes it easier to remove, as compared to the potential bleeding problem in existing cavernous hemangioma removal. ${ }^{3}$

Cavernous hemangiomas are comprised of large endothelium-lined vascular spaces. The thrombus within these vascular spaces of cavernous hemangiomas can occasionally calcify and can be identified as phlebolith on a CT Scan. Cavernous hemangiomas located in the nose or paranasal sinuses are uncommon. They are known to arise from the inferior turbinate, vomer, perpendicular plate of the ethmoid and maxillary sinus. ${ }^{4}$ It is very essential to differentiate nasal mucosal hemangiomas and hemangiomas that arise from the nasal bones or maxilla, which are primary osseous lesions, the symptoms and surgical approach of which are completely different.

Mean age at presentation of cavernous hemangiomas

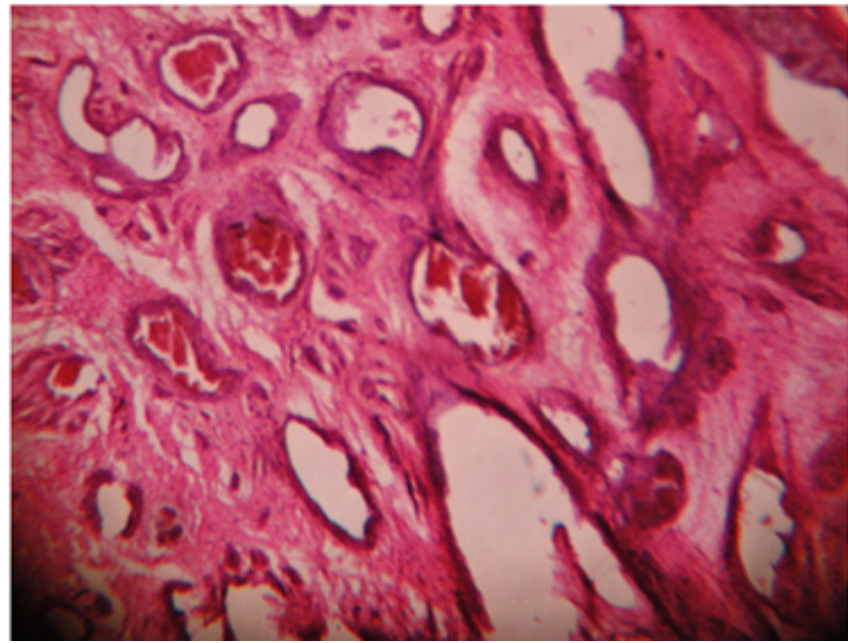

Fig. 5. Hematoxylin and eosin (H\&E, 40X).

of the nasal cavity is 40 years and with equal sex incidence. The lesion is a slowly growing, unilateral, hemorrhagic mass, frequently red or purple, which is sometimes coated with necrotic tissues. Painless growth is a characteristic symptom of nasal hemangiomas. When symptomatic, this tumor produces recurrent epistaxis or haemoptysis and nasal obstruction. ${ }^{5}$ In our case, patient presented with only nasal obstruction of both sides with no other complaints.

The differential diagnosis of hemangiomas of the nasal cavity includes inverted papilloma, olfactory neuroblastoma, lymphoma, haemangiopericytoma, haemangioendothelioma, arteriovenous fistula, lymphangioma, glomangioma, melanoma, adenocarcinoma, squamous cell carcinoma and metastatic malignancies such as renal cell carcinoma.

The pre-operative biopsy of the tumor in order to obtain a histo-pathological diagnosis is risky and must be performed with great care to avoid severe bleeding. Imaging investigations should be performed prior to any attempt of biopsy. The definitive diagnosis is made by histopathological examination of surgical specimen.

CT imaging features of the cavernous hemangioma usually shows a soft tissue density of circumscribed mass, enhancing after injection of contrast. Contrast scanning usually determines exact anatomical location and extension of the tumor. The underlying bone is usually normal but remodeling of adjacent 
surrendering structures may be seen with long-standing pressure from the expanding mass. The occurrence of phlebolith is considered to be more typical of cavernous hemangiomas. We performed non contrast CT scan because we never suspected mass to be cavernous hemangioma presenting bilaterally being extremely rare entity and not reported in literature.

MR images usually show the absence of clotted blood, which results in low signal intensity on T1weighted images and very high signal intensity on T2weighted images. Hypointense signals may represent foci of phlebolith. These lesions demonstrated little or no enhancement as they do not contain large vessels within, which is typical of other vascular malformations. ${ }^{6}$

If there is any suspicion concerning the nature of these vascular tumors, the risk of bleeding is high and angiography is always warranted, not only for diagnosis but also for pre-operative trans-arterial embolization. In the case we did not perform angiography because we never suspected that lesion was vascular. The diagnosis was a histopathological diagnosis post operatively. ${ }^{7}$

Many forms of treatment have been advocated to treat hemangiomas which includes surgical resection of the tumor, with a cuff of surrounding uninvolved tissue and ligation or cautery of the feeding surrounding vessels, being the most successful which we followed in our case. Other methods of treatment, including cryotherapy, sclerosing agent injections, corticosteroid treatment, and resection using YAG laser have been used with differing results. An alternative form of management is embolization of the hemangioma, however it may not reduce the mass completely and symptoms may persist due to mass effect.

The choice of the treatment modality is mainly surgery. The surgical approach depends on the exact location and extent of the tumor. Many surgical approaches have been suggested including the lateral rhinotomy, midfacial degloving, trans-palatal, trans-antral approach and the Le Fort I osteotomy procedure. The trans-nasal endoscopic approach has been proposed as the technique of choice in cases of intra-nasal haemangiomas of the nasal cavity and paranasal sinuses. ${ }^{8}$

In our patient, the minimally invasive trans-nasal endoscopic technique has proven to be reliable in terms of direct, fast with adequate exposure and visualization of the lesion. Because of controlled hypotensive anesthesia and cauterizing base of lesion, bleeding intraoperatively was minimal. However post operative massive bleeding required pressure anterior and posterior packing along with blood transfusion to control of bleeding. Tumor was completely removed and patient followed up six monthly for three years without recurrence. Main aim of this paper is to highlight the rare presentation of bilateral cavernous hemangioma at an unusual site with its management.

\section{Acknowledgement}

We thank erstwhile HOD and Brig Sunita Kakkar, Dept of Pathlology, Armed Forces Medical Science (AFMC) and Lt Col Vandana Rana for providing histopathology reporting and images.

\section{References}

1. Young K, Stearns G, Davidson TM. Hemangioma of the ethmoid sinus. Otolaryngol Head Neck Surg. 2000; 123:517-9

2. Genc S, Kurkcuoglu $S$ et al. Giant lobular capillary haemangioma of the nasal septum. Turk J Med Sci. 2009; 39 (2):325-8

3. Batsakis JG, Rice DH. The pathology of head and neck tumors: Vasoformative tumors, part 9A. Head Neck Surg. 1981; 3:231-9

4. Webb CG, Porter G, Sissons GRJ. Cavernous hemangioma of the nasal bones: An alternative management option. J Laryngol Otol. 2000; 114:287-9

5. Iwata $\mathrm{N}$, Hattori $\mathrm{K}$, Tsujimura $\mathrm{T}$. Hemangioma of the nasal cavity: A clinicopathological study. Auris Nasus Larynx 2002; 29:335-9

6. Dillon WP, Som PM, Rosenau W. Hemangioma of the nasal vault: MR and CT features. Radiology 1991; 180:761-5

7. Kim HJ, Kim JH, Kim JH, Hwang EG. Bone erosion caused by sinonasal cavernous hemangioma: CT findings in two patients. AJNR 1995; 16:1176-8

8. Iwata $\mathrm{N}$, Hattori $\mathrm{K}$, Tsujimura $\mathrm{T}$. Hemangioma of the nasal cavity: A clinicopathological study. Auris Nasus Larynx 2002; 29:335-9. 